\title{
THE SEMIPRIMITIVITY PROBLEM FOR GROUP ALGEBRAS OF LOCALLY FINITE GROUPS
}

\author{
D. S. Passman \\ University of Wisconsin-Madison \\ Dedicated to the memory of Professor S. A. Amitsur
}

\begin{abstract}
Let $K[G]$ be the group algebra of a locally finite group $G$ over a field $K$ of characteristic $p>0$. If $G$ has a locally subnormal subgroup of order divisible by $p$, then it is easy to see that the Jacobson radical $\mathcal{J} K[G]$ is not zero. Here, we come tantalizingly close to a complete converse by showing that if $G$ has no nonidentity locally subnormal subgroups, then $K[G]$ is semiprimitive. The proof of this theorem uses the much earlier semiprimitivity results on locally finite, locally $p$-solvable groups, and the more recent results on locally finite, infinite simple groups. In addition, it uses the beautiful properties of finitary permutation groups.
\end{abstract}

\section{$\S 1$ INTRODUCTION}

The semiprimitivity problem for group algebras $K[G]$ was intensively studied in the 1970's (see [P7]), but a good deal remains to be done. In [P2, Section I], the problem was essentially split into two parts. The first part concerns finitely generated groups, and still appears to be a rather hopeless task. The second part concerns locally finite groups, and this is much more promising. Indeed, for the remainder of this paper, we restrict our attention to the locally finite case.

In [P3], probably the last paper written on semiprimitivity until quite recently, the case of locally finite, locally $p$-solvable groups was settled. At that time, it appeared that the Classification of Finite Simple Groups (CFSG) would be required to deal with more general locally finite groups, and this has indeed proved to be the case. In the last few years, consequences of CFSG have been used in [P4], [P5], $[\mathrm{P} 6]$, and [PZ] to study the semiprimitivity of group algebras of locally finite groups under certain global assumptions. It is now time to return to the more important local assumptions.

1991 Mathematics Subject Classification. Primary: 16S34; Secondary 16N20, 20F50, 20F24.

Key words and phrases. Group algebras, semiprimitivity, locally finite groups.

Research supported in part by NSF Grant DMS-9224662.

Typeset by $\mathcal{A} \mathcal{M} \mathcal{S}-\mathrm{TEX}_{\mathrm{E}}$ 
In order to precisely state our main result, we first require some definitions and notation. Let $G$ be a locally finite group and let $K[G]$ denote the group algebra of $G$ over the field $K$. If $\mathcal{J} K[G]$ is the Jacobson radical of this algebra, then we say that $K[G]$ is semiprimitive provided $\mathcal{J} K[G]=0$. The following key observation is due to Prof. S. A. Amitsur [Am].

Lemma 1.1. If $G$ is an arbitrary group and $H$ is a subgroup of $G$, then

$$
\mathcal{J} K[G] \cap K[H] \subseteq \mathcal{J} K[H] .
$$

This result turns out to be extremely powerful because it allows us to restrict our attention to certain subgroups of $G$. For example, in the context of locally finite groups, it yields [P2, Lemma 7.4.2], namely

Lemma 1.2. $\mathcal{J} K[G]$ is a nil ideal and $K[G]$ is semiprimitive when $\operatorname{char} K=0$ or when $\operatorname{char} K=p>0$ and $G$ is a $p^{\prime}$-group.

In view of the above, it suffices to assume that char $K=p>0$, where $p$ is a prime fixed throughout the remainder of this paper. Note that a finite subgroup $A$ of $G$ is said to be locally subnormal, written $A$ lsn $G$, if $A \triangleleft \triangleleft B$ for all finite subgroups $B$ of $G$ containing $A$. For example, if $G$ is locally nilpotent, then every finite subgroup of $G$ is locally subnormal. Furthermore, every finite subnormal subgroup of $G$ is certainly locally subnormal. As we will see, if $A$ lsn $G$, then $\mathcal{J} K[A] \subseteq \mathcal{J} K[G]$, and hence, if $|A|$ is divisible by $p$, then $K[G]$ is not semiprimitive. The conjecture for locally finite groups is that this simple fact actually characterizes when $K[G]$ is semiprimitive. In other words, we suspect that $K[G]$ is semiprimitive if and only if no such $A$ exists. This has been verified for locally $p$-solvable groups in [P3] and for other families of groups in [P6]. Here, we come tantalizingly close to a complete converse with

Theorem 1.3. Let $G$ be a locally finite group and let char $K=p>0$. If $G$ has no nonidentity locally subnormal subgroups, then $K[G]$ is semiprimitive.

This is our main result and its proof will occupy the remainder of the paper. As an immediate consequence of the above, we have

Corollary 1.4. Let $G$ be a locally finite group. Then the group algebra $F[G]$ is semiprimitive for all fields $F$ of all characteristics if and only if $G$ has no nonidentity locally subnormal subgroups.

We mention this fact, not because it has any additional content, but rather because it shows that we are on the right track.

A number of the ideas in this manuscript originated during two trips I took in the summer of 1994. I would like to thank Miguel Ferrero and Said Sidki, my hosts in Brazil, for their kind hospitality, and (the late) Brian Hartley and Gary Seitz for inviting me to attend the NATO conference in Turkey on locally finite groups. I would also like to thank Jon Hall and Dick Phillips for their wonderfully informative series of talks in Istanbul on finitary locally finite groups. 


\section{$\S 2$. The Subnormal Closure}

This section contains some preliminary observations of a general nature. Recall that $G$ is a locally finite group, $K$ is a field of characteristic $p>0$, and $K[G]$ is the group algebra of $G$ over $K$.

Lemma 2.1. Let $A$ be a subgroup of $G$.

i. If $A \triangleleft \triangleleft G$, then $\mathcal{J} K[A] \subseteq \mathcal{J} K[G]$.

ii. If $A$ lsn $G$, then $\mathcal{J} K[A] \subseteq \mathcal{J} K[G]$. In particular, if $|A|$ is divisible by $p$, then $K[G]$ is not semiprimitive.

Proof. (i) If $A \triangleleft G$, then [P2, Theorem 7.2.10] implies that $\mathcal{J} K[A] \subseteq \mathcal{J} K[G]$ since $G / A$ is locally finite. The subnormal case follows easily by induction.

(ii) Now let $A$ lsn $G$, so that $A$ is a finite subgroup of $G$. If $B$ is any finite subgroup of $G$ containing $A$, then $A \triangleleft \triangleleft B$ and hence $\mathcal{J} K[A] \subseteq \mathcal{J} K[B]$ by (i). In particular, $\mathcal{J} K[A] \cdot K[B]$ is nil and therefore $\mathcal{J} K[A] \cdot K[G]$ is a nil right ideal of $K[G]$. Thus $\mathcal{J} K[A] \cdot K[G] \subseteq \mathcal{J} K[G]$, as required. Finally, if $|A|$ is divisible by $p$, then $\mathcal{J} K[A] \neq 0$ by $[\mathrm{P} 2$, Theorem 2.4.2] and hence $\mathcal{J} K[G] \neq 0$.

As a consequence, we can now offer the

Proof of Corollary 1.4. If $G$ has no nonidentity locally subnormal subgroups, then Lemma 1.2 and Theorem 1.3 imply that $F[G]$ is semiprimitive for all fields $F$ of any characteristic. Conversely, if $G$ has a nonidentity locally subnormal subgroup $A$, then we can choose a prime $q$ dividing $|A|$ and we let $\operatorname{char} F=q$. In this situation, part (ii) of the previous lemma implies that $\mathcal{J} F[G] \neq 0$, and the result follows.

Of course, we must still prove Theorem 1.3, and that is the goal of the remainder of this paper. Our argument will use a number of the basic properties of subnormal subgroups of finite groups as can be found, for example, in [W1] and [Z]. To start with, let $H \subseteq X$ be finite. Since the set of subnormal subgroups of $X$ is closed under intersection, it follows that there is a unique smallest subnormal subgroup $S$ of $X$ which contains $H$. This is called the subnormal closure of $H$ in $X$, and we denote it by $S=H^{[X]}$. If $H^{S}$ is the normal closure of $H$ in $S$, then $H \subseteq H^{S} \triangleleft S \triangleleft \triangleleft X$, so the minimal nature of $S$ implies that $S=H^{S}$. In fact, $S$ is characterized by the two properties
i. $H \subseteq S \triangleleft \triangleleft X$, and
ii. $S=H^{S}$

since (ii) implies that $H$ cannot be contained in a proper normal subgroup of $S$, and hence it is not in a proper subnormal subgroup of $S$. Note that, if $\bar{X}$ is a homomorphic image of $X$, and if $H$ and $S$ are as above, then $\bar{H} \subseteq \bar{S} \triangleleft \triangleleft \bar{X}$ and $\bar{S}=\bar{H}^{\bar{S}}$. Thus $\bar{S}$ is the subnormal closure of $\bar{H}$ in $\bar{X}$. In general, subnormal closures do not exist for arbitrary subgroups of infinite groups. 
Now if $H \subseteq X \subseteq Y$ are all finite, then $H \subseteq H^{[Y]} \cap X \triangleleft \triangleleft X$. Thus the minimal nature of $H^{[\bar{X}]}$ implies that $H^{[X]} \subseteq H^{[Y]} \cap X \subseteq H^{[Y]}$. As will be apparent, this inclusion allows us to define a local subnormal closure for finite subgroups of locally finite groups, and we use the same notation. Specifically, if $H$ is a finite subgroup of $G$, then we set

$$
H^{[G]}=\bigcup_{X} H^{[X]}
$$

where the union is over all finite subgroups $X$ of $G$ containing $H$. Note that, if $G$ is finite, then the inclusion $H^{[X]} \subseteq H^{[Y]}$ immediately implies that the two possible meanings for $H^{[G]}$ are, in fact, the same.

At times, it will be necessary for us to work in the context of a fixed set of generating subgroups for $G$. Recall that $\mathcal{L}$ is said to be a local system for $G$ if $\mathcal{L}$ is a collection of finite subgroups with the property that every finite subgroup $H$ of $G$ is contained in some $L \in \mathcal{L}$. In particular, if $L_{1}, L_{2} \in \mathcal{L}$, then there exists some $L \in \mathcal{L}$ with $\left\langle L_{1}, L_{2}\right\rangle \subseteq L$, and this property, along with $G=\langle L \mid L \in \mathcal{L}\rangle$, clearly characterizes a local system.

Lemma 2.2. Let $H$ be a finite subgroup of $G$, and set $S=H^{[G]}$.

i. $S$ is a subgroup of $G$ containing $H$.

ii. If $\mathcal{L}$ is a local system for $G$, then $\left\{H^{[L]} \mid L \in \mathcal{L}, L \supseteq H\right\}$ is a local system for the subgroup $S$.

iii. If $A$ lsn $S$, then $A \operatorname{lsn} G$.

iv. $S=H^{S}$ is the normal closure of $H$ in $S$, and $\mathbb{N}_{G}(H) \subseteq \mathbb{N}_{G}(S)$.

Proof. (i) If $X$ and $Y$ are finite subgroups of $G$ containing $H$ and if $Z=\langle X, Y\rangle$, then $\left\langle H^{[X]}, H^{[Y]}\right\rangle \subseteq H^{[Z]} \subseteq H^{[G]}$. Thus $H^{[G]}$ is a subgroup of $G$ with the set of all such $H^{[X]}$ as a local system.

(ii) Let $X$ be a finite subgroup of $S$. Then (i) implies that $X \subseteq H^{[Y]}$ for some finite subgroup $Y$ of $G$ containing $H$. Furthermore, since $\mathcal{L}$ is a local system for $G$, there exists $L \in \mathcal{L}$ with $Y \subseteq L$. Thus $X \subseteq H^{[Y]} \subseteq H^{[L]} \subseteq H^{[G]}$, as required.

(iii) Let $A$ lsn $S$ and, by (i), let $X$ be a finite subgroup of $G$ containing $H$ with $A \subseteq H^{[X]}$. Now let $B$ be any finite subgroup of $G$ containing $X$. Then $A \subseteq H^{[\bar{X}]} \subseteq H^{[B]} \subseteq H^{[G]}$, so $A \triangleleft \triangleleft H^{[B]}$ since $A$ lsn $S$. But $H^{[B]} \triangleleft \triangleleft B$, so we conclude that $A \triangleleft \triangleleft B$, and hence $A$ lsn $G$.

(iv) The equality $S=H^{S}$ is clear since $H^{S}$ contains each $H^{[X]}$. For the remaining part, let $g \in \mathbb{N}_{G}(H)$ and let $Y$ be any finite subgroup of $G$ containing $g$ and $H$. Then $H \subseteq H^{[Y]}$ implies that $H=g^{-1} H g \subseteq g^{-1} H^{[Y]} g$. But the latter group is also subnormal in $Y$, so $g^{-1} H^{[Y]} g=H^{[Y]}$ and it follows that $g$ normalizes $S=H^{[G]}=\bigcup_{Y} H^{[Y]}$.

We remark that parts (ii) and (iii) above allow us to reduce the proof of Theorem 1.3 to certain local subnormal closures. However, it is the condition $S=H^{S}$ in (iv) which really turns out to be crucial. 
If $N$ is any subgroup of $G$, we define its almost or finitary centralizer by

$$
\mathbb{D}_{G}(N)=\left\{x \in G|| N: \mathbb{C}_{N}(x) \mid<\infty\right\},
$$

and it follows easily that $\mathbb{D}_{G}(N)$ is a subgroup of $G$. Furthermore, if $N \triangleleft G$, then $\mathbb{D}_{G}(N)$ is the normal subgroup of $G$ consisting of those elements which act in a finitary manner on $N$. Note that

$$
\mathbb{D}_{G}(G)=\Delta(G)=\left\{x \in G|| G: \mathbb{C}_{G}(x) \mid<\infty\right\},
$$

and this group is called the finite conjugate or f.c. center of $G$ since it consists of those elements belonging to finite conjugacy classes. We say that $G$ is an f.c. group if $G=\Delta(G)$ and, in the context of locally finite groups, this is equivalent to $G$ being locally normal. In other words, $G=\Delta(G)$ if and only if every finite subgroup of $G$ is contained in a finite normal subgroup (see [P2, Lemma 4.1.8]). In fact, if $G$ is an arbitrary periodic group, then $\Delta(G)$ is generated by, and hence is the join of, the finite normal subgroups of $G$.

For any subgroup $D$ of $G$ there is a natural $K[D]$-bimodule projection map $\pi_{D}: K[G] \rightarrow K[D]$ given by

$$
\pi_{D}: \sum_{x \in G} a_{x} x \mapsto \sum_{x \in D} a_{x} x
$$

Thus $\pi_{D}$ is the linear extension of the map $G \rightarrow D \cup\{0\}$ which is the identity on $D$ and zero on $G \backslash D$. The following key observation is essentially [P3, Lemma 3.7]. Its proof uses basic properties of the Jacobson radical of $K[G]$ as well as the known structure of the nilpotent radical of the group algebra.

Lemma 2.3. If $N \triangleleft G$ with $\mathcal{J} K[N]=0$, then $\mathcal{J} K[G]=\mathcal{J} K[D] \cdot K[G]$ where $D=\mathbb{D}_{G}(N)$. In particular,

$$
\pi_{D}(\mathcal{J} K[G])=\mathcal{J} K[D] \subseteq \mathcal{J} K[G] .
$$

If $\alpha=\sum a_{x} x \in K[G]$, then the support of $\alpha$ is the finite subset of $G$ given by $\operatorname{supp} \alpha=\left\{x \in G \mid a_{x} \neq 0\right\}$, and we call $H=\langle\operatorname{supp} \alpha\rangle$ the supporting subgroup of $\alpha$. Clearly $H$ is the smallest subgroup of $G$ with $\alpha \in K[H]$ and, since $G$ is locally finite, $H$ is finite. Next, we say that $\beta \in K[G]$ is a truncation of $\alpha$ if $\beta=\sum^{\prime} a_{x} x$, where $\sum^{\prime}$ indicates a partial sum of the terms in $\alpha$. In addition, $\beta$ is a proper truncation if $\beta \neq 0$ or $\alpha$. The next lemma is our first application of the subnormal closure. 
Lemma 2.4. Let $G$ be a locally finite group with local system $\mathcal{L}$. Suppose $\mathcal{J} K[G] \neq$ 0 and choose $0 \neq \alpha \in \mathcal{J} K[G]$ of minimal nonzero support size. We can assume that $1 \in \operatorname{supp} \alpha$ and we set $H=\langle\operatorname{supp} \alpha\rangle$. Then there exists a finite subgroup $W$ of $G$ containing $H$ such that

i. $W \triangleleft \triangleleft L$ for some $L \in \mathcal{L}$,

ii. $W=H^{W}$ is the normal closure of $H$ in $W$,

iii. no proper truncation of $\alpha$ is contained in $\mathcal{J} K[W]$, and

iv. if $N$ is any subgroup of $G$ normalized by $W$ and satisfying $\mathcal{J} K[N]=0$, then $W$ is contained in $\Delta(N W)$.

Proof. If $\alpha$ is a nonzero element of $\mathcal{J} K[G]$ of minimal support size and if $g \in \operatorname{supp} \alpha$, then $g^{-1} \alpha \in \mathcal{J} K[G], 1 \in \operatorname{supp} g^{-1} \alpha$, and $\left|\operatorname{supp} g^{-1} \alpha\right|=|\operatorname{supp} \alpha|$. Thus, by replacing $\alpha$ by $g^{-1} \alpha$ if necessary, we can assume that $1 \in \operatorname{supp} \alpha$.

Let $\beta_{1}, \beta_{2}, \ldots, \beta_{k}$ be the finitely many proper truncations of $\alpha$. Since $0<$ $\left|\operatorname{supp} \beta_{i}\right|<|\operatorname{supp} \alpha|$, the minimal nature of $|\operatorname{supp} \alpha|$ implies that no $\beta_{i}$ is contained in $\mathcal{J} K[G]$. In particular, the right ideals $\beta_{i} K[G]$ are not nil, so there exist elements $\gamma_{i} \in K[G]$ with $\beta_{i} \gamma_{i}$ not nilpotent. Since $\mathcal{L}$ is a local system for $G$, there exists $L \in \mathcal{L}$ containing $H$ and the supports of all the $\gamma_{i}$. Because $\mathcal{J} K[L]$ is nilpotent, it follows that $\beta_{i} \notin \mathcal{J} K[L]$ for all $i$.

Now let $W=H^{[L]}$ be the subnormal closure of $H$ in $L$. Then certainly (i) and (ii) above are satisfied. Furthermore, since $W \triangleleft \triangleleft L$, it follows from Lemma 2.1(i) that $\mathcal{J} K[W] \subseteq \mathcal{J} K[L]$. Thus $\beta_{i} \notin \mathcal{J} K[L]$ implies $\beta_{i} \notin \mathcal{J} K[W]$, and (iii) is proved.

For part (iv), suppose $N$ is any subgroup of $G$ normalized by $W$ and satisfying $\mathcal{J} K[N]=0$. If $X=N W$, then $N \triangleleft X$, so the previous lemma implies that $\pi_{D}(\mathcal{J} K[X]) \subseteq \mathcal{J} K[X]$, where $D=\mathbb{D}_{X}(N) \triangleleft X$. Thus, since $\alpha \in \mathcal{J} K[G] \cap K[X] \subseteq$ $\mathcal{J} K[X]$ by Lemma 1.1 , we have $\pi_{D}(\alpha) \in \mathcal{J} K[X] \cap K[W] \subseteq \mathcal{J} K[W]$. But $\pi_{D}(\alpha)$ is a truncation of $\alpha$ which is not zero since $1 \in \operatorname{supp} \alpha$. Thus part (iii) implies that $\pi_{D}(\alpha)=\alpha$ and hence that $H=\langle\operatorname{supp} \alpha\rangle \subseteq D$. Indeed, since $D \triangleleft X$, (ii) yields $W=H^{W} \subseteq D$. Finally, $|X: N| \leq|H|<\infty$, so it is clear that $D=\Delta(X)$, and therefore $W \subseteq \Delta(X)$, as required.

We close this section with a routine result which allows us to reduce the proof of Theorem 1.3 to the case of countable groups. Such a reduction makes the verification of the main theorem somewhat easier to understand.

Lemma 2.5. Let $*$ be a property of finite groups (like being solvable or having order divisible by $p$ ) and let $G$ be a locally finite group having no locally subnormal *-subgroups. Let $\mathcal{L}$ be a local system for $G$ and let $H$ be a countable subgroup of $G$. Then there exists a subgroup $\tilde{H}$ of $G$ such that

i. $\tilde{H} \supseteq H$ and $\tilde{H}$ is countable,

ii. $\tilde{H}$ has no locally subnormal $*$-subgroups,

iii. $\{L \in \mathcal{L} \mid L \subseteq \tilde{H}\}$ is a local system for $\tilde{H}$. 
Hence, by (i) and (iii), $\tilde{H}$ is the ascending union of groups $L_{1} \subseteq L_{2} \subseteq L_{3} \subseteq \cdots$ with each $L_{i}$ in $\mathcal{L}$.

Proof. We construct an ascending series $H=H_{0} \subseteq H_{1} \subseteq H_{2} \subseteq \cdots$ of countable groups satisfying

1. $H_{i}$ is the ascending union of the finite groups $L_{i, 1} \subseteq L_{i, 2} \subseteq L_{i, 3} \subseteq \cdots$ with $L_{i, j} \in \mathcal{L}$ when $i \neq 0$, and

2. no finite $*$-subgroup of $H_{i-1}$ is locally subnormal in $H_{i}$.

To start with, since $H=H_{0}$ is countable, it follows that $H_{0}$ is an ascending union of finite subgroups $L_{0,1} \subseteq L_{0,2} \subseteq L_{0,3} \subseteq \cdots$. Note that these subgroups are not assumed to belong to $\mathcal{L}$.

Now suppose that $H_{i}=\bigcup_{j} L_{i, j}$ is given. Since $H_{i}$ is countable, it has a countable number of finite $*$-subgroups, say $A_{i, 1}, A_{i, 2}, A_{i, 3}, \ldots$ By assumption, $A_{i, j}$ is not locally subnormal in $G$, and hence there exists a finite subgroup $B_{i, j}$ of $G$ with $A_{i, j}$ not subnormal in $B_{i, j}$. We now choose the groups $L_{i+1, j} \in \mathcal{L}$ inductively so that $L_{i+1, j}$ contains $L_{i+1, j-1}, B_{i, j}$, and $L_{i, j}$. Since $L_{i+1, j} \supseteq L_{i+1, j-1}$, it follows that $H_{i+1}=\bigcup_{j} L_{i+1, j}$ is a countable subgroup of $G$. Furthermore, $L_{i+1, j} \supseteq L_{i, j}$ and $H_{i}=\bigcup_{j} L_{i, j}$, so $H_{i+1} \supseteq H_{i}$. Finally, $A_{i, j}$ is not subnormal in $B_{i, j} \subseteq H_{i+1}$, so $A_{i, j}$ is not locally subnormal in $H_{i+1}$. Thus conditions (1) and (2) are satisfied by $H_{i+1}$.

It follows that the sequence $H=H_{0} \subseteq H_{1} \subseteq H_{2} \subseteq \cdots$ exists, and we set $\tilde{H}=\bigcup_{i} H_{i}$. Certainly, $\tilde{H} \supseteq H$ and $\tilde{H}$ is countable, so (i) holds. Furthermore, the set $\left\{L_{i, j} \mid i \neq 0\right\}$ is a local system for $\tilde{H}$ contained in $\mathcal{L}$ since any finite subgroup of $\tilde{H}$ is contained in some $H_{i}$ with $i \neq 0$. This proves (iii) and the same remark, along with condition (2), yields property (ii). The final observation, namely that $\tilde{H}$ is an ascending union of members of $\mathcal{L}$, is now obvious.

\section{§3. Locally Solvable-By-Semisimple Groups}

As we mentioned earlier, consequences of the Classification of Finite Simple Groups were used to study the semiprimitivity of group algebras of locally finite groups under certain global assumptions. For example, [P4] and [PZ] handled the infinite simple linear and nonlinear groups, respectively, while [P5] and [P6] used these results to handle groups with certain types of finite subnormal series. The following is a slight generalization of the main theorem of [P6] merged with the main result of [P3]. Note that the transitivity of subnormality implies that if $A \operatorname{lsn} N$ and $N \triangleleft \triangleleft G$, then $A$ lsn $G$.

Proposition 3.1. Let $K[G]$ be the group algebra of a locally finite group $G$ over a field $K$ of characteristic $p>0$. Suppose that $G$ has a finite subnormal series

$$
G_{0} \triangleleft G_{1} \triangleleft \cdots \triangleleft G_{n}=G
$$

with each quotient $G_{i+1} / G_{i}$ either

i. locally p-solvable, or 
ii. an infinite simple group, or

iii. generated by its locally subnormal subgroups.

Suppose further that $\mathcal{J} K\left[G_{0}\right]=0$. Then $K[G]$ is semiprimitive if and only if $G$ has no locally subnormal subgroups of order divisible by $p$.

Proof. In view of Lemma 2.1(ii), it suffices to show that if $G$ has no locally subnormal subgroups of order divisible by $p$, then $\mathcal{J} K[G]=0$. For this, we proceed by induction on $n$, the case $n=0$ being given. Now suppose the result holds for $n-1$ and set $H=G_{n-1} \triangleleft G$. Since $H$ has no locally subnormal subgroups of order divisible by $p$, the inductive assumption implies that $\mathcal{J} K[H]=0$. Furthermore [P6, Lemma 3.4(iii)] implies that $\mathcal{J} K[G]=0$ if $G / H=G_{n} / G_{n-1}$ is generated by its locally subnormal subgroups. Thus we can assume that $G / H$ is either locally $p$-solvable or infinite simple.

Now $\mathcal{J} K[H]=0$, so Lemma 2.3 implies that $\mathcal{J} K[G]=\mathcal{J} K[D] \cdot K[G]$ where $D=\mathbb{D}_{G}(H)$, and it suffices to show that $\mathcal{J} K[D]=0$. To this end, note that

$$
N=D \cap H=\mathbb{D}_{G}(H) \cap H=\mathbb{D}_{H}(H)=\Delta(H)
$$

is an f.c. group. Thus, since $N \triangleleft G$ has no locally subnormal subgroups of order divisible by $p$, it follows that $N$ is a $p^{\prime}$-group and hence that $\mathcal{J} K[N]=0$ by Lemma 1.2. Furthermore,

$$
D / N=D /(D \cap H) \cong D H / H \triangleleft G / H .
$$

In particular, if $G / H$ is locally $p$-solvable, then $D$ is also locally $p$-solvable and [P3, Corollary 4.6] yields the result. On the other hand, if $G / H$ is infinite simple, then either $D=N$ or $D / N$ is infinite simple. In the former case, $\mathcal{J} K[D]=\mathcal{J} K[N]=0$ as required, and thus it suffices to assume that $D / N$ is infinite simple. For this, note that $D$ acts in a finitary manner on $H$, so it certainly acts in a finitary manner on $N=H \cap D$. In other words, $D=\mathbb{D}_{D}(N)$, and therefore the result follows from [P6, Proposition 3.3].

It is interesting to compare the above to the following more elementary consequence of Lemma 2.3. Here we make the stronger assumption that $G$ has no nonidentity locally subnormal subgroups.

Lemma 3.2. Let $G$ have a finite subnormal series

$$
\langle 1\rangle=G_{0} \triangleleft G_{1} \triangleleft \cdots \triangleleft G_{n}=G
$$

and suppose that $\mathcal{J} K\left[G_{i+1} / G_{i}\right]=0$ for each $i=0,1, \ldots, n-1$. If $G$ has no nonidentity locally subnormal subgroups, then $K[G]$ is semiprimitive.

Proof. Again, we procede by induction on $n$, the case $n=1$ being given. Now suppose the result holds for $n-1$ and set $H=G_{n-1} \triangleleft G$. Since $H$ has no 
nonidentity locally subnormal subgroups, the inductive assumption implies that $\mathcal{J} K[H]=0$. Thus, by Lemma $2.3, \mathcal{J} K[G]=\mathcal{J} K[D] \cdot K[G]$, where $D=\mathbb{D}_{G}(H)$.

Now $D \cap H=\Delta(H) \triangleleft G$ is a locally normal group. Thus, since $D \cap H$ has no nonidentity locally subnormal subgroups, it follows that $D \cap H=\langle 1\rangle$. In other words, $D$ is isomorphic to a normal subgroup of $G / H=G_{n} / G_{n-1}$. But $\mathcal{J} K\left[G_{n} / G_{n-1}\right]=0$, so $\mathcal{J} K[D]=0$ by Lemma 2.1 , and therefore $\mathcal{J} K[G]=0$ as required.

The following is an immediate consequence of [BP, (4.1)] and Proposition 3.1 with $G_{0}=\langle 1\rangle$. While it is not related to the main goal of this paper, the result is of interest because it completes the solution of the semiprimitivity problem for linear groups which are not necessarily locally finite. See [P7] for details.

Corollary 3.3. Let $G$ be a locally finite linear group in any characteristic. Then $K[G]$ is semiprimitive if and only if $G$ has no locally subnormal subgroups of order divisible by $p$.

Recall that a finite group $S$ is said to be semisimple if it is a direct product of nonabelian simple groups. Here we follow the notation of [W1] and [Z], rather than that of [G]. As is well known, if $S=M_{1} \times M_{2} \times \cdots \times M_{k}$, with each $M_{i}$ nonabelian and simple, then every normal subgroup $N$ of $S$ is a partial direct product of the $M_{i}$ 's. Thus $N$ is also semisimple, and it follows easily by induction that every subnormal subgroup of $S$ is normal and hence of the above form. For convenience, we call $k$ the width of $S$, and we write $k=$ wd $S$.

Next, if $S$ is any finite group, then we let sol $S$ denote its unique largest normal solvable subgroup. Thus, sol $S$ is characteristic in $S$ and, by [Z, page 246], it contains all solvable subnormal subgroups of $S$. In particular, if $N \triangleleft \triangleleft S$, then it follows that $\operatorname{sol} N=N \cap \operatorname{sol} S$.

Finally, we say that $S$ is solvable-by-semisimple if $S / \operatorname{sol} S$ is semisimple. In this case, we define the width of $S$ to be that of $S / \operatorname{sol} S$, so that wd $S$ is the number of simple factors of the semisimple group $S / \operatorname{sol} S$. Note that, if $N \triangleleft \triangleleft S$, then

$$
N / \operatorname{sol} N=N /(N \cap \operatorname{sol} S) \cong N(\operatorname{sol} S) / \operatorname{sol} S \triangleleft \triangleleft S / \operatorname{sol} S .
$$

Thus $N / \operatorname{sol} N$ is semisimple, $N$ is solvable-by-semisimple, and wd $N \leq$ wd $S$. Furthermore, if $N \triangleleft S$, then $N(\operatorname{sol} S) / N$ is a normal solvable subgroup of $S / N$ with

$$
\frac{S / N}{N(\operatorname{sol} S) / N} \cong \frac{S}{N(\operatorname{sol} S)} \cong \frac{S / \operatorname{sol} S}{N(\operatorname{sol} S) / \operatorname{sol} S}
$$

Thus $S / N$ is also solvable-by-semisimple and $\operatorname{wd} N+\operatorname{wd} S / N=\operatorname{wd} S$.

We say that a group $G$ is locally solvable-by-semisimple if it has a local system consisting of finite solvable-by-semisimple groups. The next result is a first step towards settling the semiprimitivity problem for the group algebras of such groups. 
Lemma 3.4. Let $T=\bigcup_{i=1}^{\infty} T_{i}$ be an ascending union of the finite solvable-bysemisimple groups $T_{i}$ and assume that there is an integer $k$ such that each $T_{i}$ has width $\leq k$. Then $T$ has a finite subnormal series

$$
\langle 1\rangle=N_{0} \triangleleft N_{1} \triangleleft \cdots \triangleleft N_{r}=T
$$

such that each factor $N_{i+1} / N_{i}$ is either a simple group or locally solvable. In particular, if $T$ has no locally subnormal subgroups of order divisible by $p$, then $K[T]$ is semiprimitive.

Proof. We proceed by induction on $k$, the case $k=0$ being trivial since any such group $T$ is clearly locally solvable. Now suppose that the result holds for $k-1$ and let $T$ have parameter $k$.

We first observe that if $N \triangleleft T$, then $N=\bigcup_{i=1}^{\infty}\left(N \cap T_{i}\right)$ and each $N \cap T_{i}$ is solvable-by-semisimple with $\operatorname{wd}\left(N \cap T_{i}\right) \leq \mathrm{wd} T_{i}$. Similarly, $T / N=\bigcup_{i=1}^{\infty} T_{i} N / N$ and $T_{i} N / N \cong T_{i} /\left(N \cap T_{i}\right)$ is solvable-by-semisimple with wd $T_{i} /\left(N \cap T_{i}\right) \leq \operatorname{wd} T_{i}$. Thus, all normal subgroups and factor groups of $T$ inherit the properties of $T$, with the same parameter $k$.

Now let $A$ be the union of all locally solvable normal subgroups of $T$. Then $A$ is a locally solvable characteristic subgroup of $T$, and $T / A$ has no nonidentity locally solvable normal subgroups. Since $T / A$ has the same structure as that of $T$, it therefore suffices to consider $T / A$. In other words, we may assume that $A=\langle 1\rangle$ and hence that $T$ has no nonidentity locally solvable normal subgroups.

Next, let $B$ be the intersection of all normal subgroups $X$ of $T$ with $T / X$ locally solvable. Then $T / B$ embeds in $\prod_{X} T / X$ and, since $T / B$ is locally finite, it follows easily that $T / B$ is locally solvable. Thus $B$ is a characteristic subgroup of $T$ having no nonidentity homomorphic image which is locally solvable. Again, $B$ has the same structure as that of $T$, so it suffices to assume that $B=T$. In other words, we may now suppose that $T$ has no nontrivial locally solvable normal subgroups or factor groups.

If $T$ is simple, we are done. If not, let $N$ be a nontrivial normal subgroup of $T$. Then, as we noted before, $N=\bigcup_{i=1}^{\infty}\left(N \cap T_{i}\right)$ and $T / N \cong \bigcup_{i=1}^{\infty} T_{i} /\left(N \cap T_{i}\right)$. Furthermore,

$$
\operatorname{wd}\left(N \cap T_{i}\right)+\operatorname{wd} T_{i} /\left(N \cap T_{i}\right)=\operatorname{wd} T_{i} \leq k .
$$

Since $T / N$ is not locally solvable, we must have $\operatorname{wd} T_{i} /\left(N \cap T_{i}\right) \geq 1$ for almost all $i$. Thus $\operatorname{wd}\left(N \cap T_{i}\right) \leq k-1$ for infinitely many subscripts and, by induction, the result holds for the group $N$. Similarly, since $N$ is not locally solvable, $\operatorname{wd}\left(N \cap T_{i}\right) \geq 1$ for almost all $i$, so wd $T_{i} /\left(N \cap T_{i}\right) \leq k-1$ for infinitely many subscripts and, by induction, the result also holds for $T / N$.

In other words, both $N$ and $T / N$ have suitable finite subnormal series and, by combining these, we obtain the required series for $T$. Proposition 3.1 now yields the semiprimitivity aspect of the result. 
We now consider more general locally solvable-by-semisimple groups. Here our result is not as sharp as the above because we assume that $G$ has no nonidentity locally subnormal subgroups rather than the expected no locally subnormal subgroups of order divisible by $p$.

Let us recall some notation and several elementary lemmas from [PZ]. To start with, let $\left\{x_{1}, x_{2}, \ldots, x_{s}\right\}$ be a finite subset of $G^{\#}=G \backslash\{1\}$. Then an element $z \in G$ is said to be a $p$-insulator for this set if $z$ is a $p$-element, but $z x_{i}$ is not a $p$-element for any $i=1,2, \ldots, s$. It follows, as in [PZ, Lemma 7.4(ii)], that if $\alpha \in K[G]$ with $\operatorname{supp} \alpha=\left\{1, x_{1}, x_{2}, \ldots, x_{s}\right\}$ and if $z$ exists, then $z \alpha$ is not nilpotent and hence $\alpha \notin \mathcal{J} K[G]$. Now, it is easy to see that $p$-insulators exist for single element sets. Indeed, let $H$ be any group with $\mathbb{O}_{p}(H)=\langle 1\rangle$ and let $x \in H^{\#}$. Then [PZ, Lemma 1.4] guarantees the existence of a $p$-element $h \in H$ such that $h x$ is not a $p$-element. For sets with at least two elements, the existence of a $p$-insulator is more problematic.

Finally, let $\mathcal{N}$ denote the set of natural numbers and suppose $f_{1}, f_{2}, \ldots, f_{k}$ are maps from $\mathcal{N}$ to $\mathcal{N}$. As is well known (see for example [PZ, Lemma 1.3]), if each $f_{i}$ is unbounded on all subsequences of $\mathcal{N}$, then there exists a subsequence $\mathcal{M} \subseteq \mathcal{N}$ such that each $f_{i}$ is strictly increasing on $\mathcal{M}$. In particular, $\min _{i}\left\{f_{i}\right\}$ is unbounded on $\mathcal{M}$.

Lemma 3.5. Let $G=\bigcup_{i=1}^{\infty} L_{i}$ be the ascending union of the finite solvable-bysemisimple subgroups $L_{i}$. If $G$ has no nonidentity locally subnormal subgroups, then $K[G]$ is semiprimitive.

Proof. We suppose, by way of contradiction, that $G$ has no nonidentity locally subnormal subgroups and that $\mathcal{J} K[G] \neq 0$. Because of the latter, we can choose $\alpha, H$ and $W$ as in Lemma 2.4. In particular, $\alpha$ is a nonzero element of minimal support size in $\mathcal{J} K[G]$ with $1 \in \operatorname{supp} \alpha, H=\langle\operatorname{supp} \alpha\rangle$, and $W$ is a finite group containing $H$ with $W=H^{W}$. By deleting terms if necessary, we can assume that $W \subseteq L_{1}$ and hence that $W \subseteq L_{i}$ for all $i$.

Write $\operatorname{supp} \alpha=\left\{1, x_{1}, x_{2}, \ldots, x_{s}\right\} \subseteq H$, let $X_{j}=\left\langle x_{j}\right\rangle$, and define $T_{j}=X_{j}^{G}$ to be the normal closure of $X_{j}$ in $G$. Since $X_{j} \subseteq L_{1}$, it is clear that $T_{j}$ is the ascending union $T_{j}=\bigcup_{i} X_{j}^{L_{i}}$ and, of course, each $X_{j}^{L_{i}}$ is solvable-by-semisimple. We can now let $f_{j}(i)$ be the width of $X_{j}^{L_{i}}$ and, in this way, we obtain finitely many functions $f_{1}, f_{2}, \ldots, f_{s}: \mathcal{N} \rightarrow \mathcal{N}$. There are two cases to consider according to whether the $f_{j}$ 's are bounded or not.

Case 1. Assume that some $f_{j}$ is bounded on a subsequence of $\mathcal{N}$.

Proof. By deleting terms, we can assume that $f_{j}$ is bounded on $\mathcal{N}$. For convenience, write $X=X_{j}$ and $T=T_{j}=X^{G}$. Then $T$ is the ascending union of the solvableby-semisimple groups $X^{L_{i}}$, each of width at most $\max f_{j}(\mathcal{N})$. Furthermore, since $T \triangleleft G, T$ has no nonidentity locally subnormal subgroups. Thus, by Lemma 3.4, $\mathcal{J} K[T]=0$. Furthermore, since $W$ normalizes $T \triangleleft G$, we can apply Lemma 2.4(iv) 
with $N=T$ to conclude that $W \subseteq \Delta(T W)$ and therefore that $X \subseteq T \cap \Delta(T W) \subseteq$ $\Delta(T)$. But $\Delta(T)$ is generated by all finite normal subgroups of $T$, so $\Delta(T)=\langle 1\rangle$ and this is a contradiction since $X \neq\langle 1\rangle$.

Case 2. Assume that the $f_{j}$ are unbounded on all subsequences of $\mathcal{N}$.

Proof. Here we construct a $p$-insulator for the set $\left\{x_{1}, x_{2}, \ldots, x_{s}\right\}$. To this end, note that $\min _{j}\left\{f_{j}\right\}$ is an unbounded function on $\mathcal{N}$, and hence there exists $n \in \mathcal{N}$ with $f_{j}(n) \geq s$ for all $j$. Write $L_{n} / \operatorname{sol} L_{n}=M_{1} \times M_{2} \times \cdots$ as a finite direct product of nonabelian simple groups, and let $\pi_{k}: L_{n} \rightarrow M_{k}$ and $\pi: L_{n} \rightarrow L_{n} / \operatorname{sol} L_{n}$ be the natural epimorphisms.

Since $\left\langle x_{j}\right\rangle=X_{j} \subseteq L_{n}$, it is clear that $\pi\left(X_{j}^{L_{n}}\right)=\pi\left(X_{j}\right)^{\pi\left(L_{n}\right)}$ is the direct product of those $M_{k}$ 's with $\pi_{k}\left(x_{j}\right) \neq 1$. In particular, since $f_{j}(n) \geq s$, it follows that each $x_{j}$ projects nontrivially to at least $s$ of the factors $M_{k}$. But there are only $s$ members of the set $\left\{x_{1}, x_{2}, \ldots, x_{s}\right\}$, and therefore it is obvious that we can relable the $M_{k}$ 's in such a way that $\pi_{j}\left(x_{j}\right) \neq 1$ for all $j=1,2, \ldots, s$.

Finally, since $\mathbb{O}_{p}\left(M_{j}\right)=\langle 1\rangle$, [PZ, Lemma 1.4] guarantees the existence of a $p$-element $\bar{z}_{j} \in M_{j}$ with $\bar{z}_{j} \pi_{j}\left(x_{j}\right)$ not a $p$-element, and we set $\bar{z}=\bar{z}_{1} \bar{z}_{2} \cdots \bar{z}_{s} \in$ $L_{n} / \operatorname{sol} L_{n}$. Then $\bar{z}$ is obviously a $p$-element, and hence we can choose a $p$-element $z \in L_{n} \subseteq G$ with $\pi(z)=\bar{z}$. Now note that $\pi_{j}\left(z x_{j}\right)=\pi_{j}(z) \pi_{j}\left(x_{j}\right)=\bar{z}_{j} \pi_{j}\left(x_{j}\right)$ is not a $p$-element for any $j$. Thus $z x_{j}$ is not a $p$-element, and $z$ is indeed a $p$-insulator for the set $\left\{x_{1}, x_{2}, \ldots, x_{s}\right\}$. But $\operatorname{supp} \alpha=\left\{1, x_{1}, x_{2}, \ldots, x_{s}\right\}$, so [PZ, Lemma 7.4(ii)] implies that $\alpha \notin \mathcal{J} K[G]$, and this contradiction yields the result.

\section{§4. Finitary Permutation Groups}

If $\Omega$ is any nonempty set, we let $\operatorname{Sym}_{\Omega}$ denote the full group of permutations on $\Omega$, and we let $\mathrm{FSym}_{\Omega}$ denote its normal subgroup consisting of all those permutations which move only finitely many points. Thus the finitary symmetric group FSym $_{\Omega}$ is a locally finite group having the finitary alternating group FAlt $\Omega$ as a normal subgroup of index 2. If $|\Omega| \geq 5$, then FAlt $\Omega$ is of course a simple group and, for

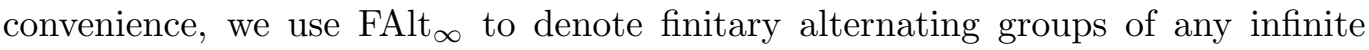
size. The following result is an immediate consequence of the work of [W2] and is presumably well known to the experts.

Proposition 4.1. Let $G$ be a permutation group on the set $\Omega$ and let $H$ be a finite subgroup of $G$ which moves only finitely many points. If $G=H^{G}$ is the normal closure of $H$ in $G$, then $G$ has a finite subnormal series with factors which are either locally finite f.c. groups or isomorphic to suitable FAlt $_{\infty}$.

Proof. By assumption, $G \subseteq \operatorname{Sym}_{\Omega}$ and $H \subseteq \operatorname{FSym}_{\Omega} \triangleleft \operatorname{Sym}_{\Omega}$. Thus since $G=H^{G}$, it follows that $G \subseteq$ FSym $_{\Omega}$ and hence that $G$ is locally finite. Furthermore, since $H$ can act nontrivially on only finitely many orbits, the same is true of $G$. Thus, by deleting one point orbits if necessary, we can assume that $G$ has only finitely many orbits, say $\Omega_{1}, \Omega_{2}, \ldots, \Omega_{k}$. We proceed by induction on $k$. 
Case 1. $k=1$

Proof. By assumption, $G$ is transitive on $\Omega=\Omega_{1}$. If $|\Omega|<\infty$, then $G$ is a finite group and hence an f.c. group. Thus we may suppose that $\Omega$ is infinite and that $H$ moves precisely $t$ points.

If $\Lambda$ is a block of imprimitivity and if $|\Lambda|>t$, then $H$ fixes a point in $\Lambda$ and hence $H$ stabilizes $\Lambda$. Furthermore, since any $G$-conjugate of $H$ also moves precisely $t$ points, it follows that $\Lambda$ is stabilized by all such $H^{g}$. But $G=H^{G}$ is generated by the conjugates of $H$, so $\Lambda$ is stabilized by $G$ and hence $\Lambda=\Omega$. In other words, all proper blocks have size $\leq t$, and therefore we can choose $\Gamma$ to be a proper block of maximal size.

Now, the maximality of $\Gamma$ clearly implies that $G$ is primitive on the set of blocks $\bar{\Omega}=\{\Gamma g \mid g \in G\}$. Hence, if $N$ is the kernel of the action of $G$ on $\bar{\Omega}$, then $\bar{G}=G / N \cong$ FSym $_{\infty}$ or FAlt $\infty$ by [W2, Satz 9.4] (or see [W3, page 228]). On the other hand, $N$ stabilizes each $\Gamma g$ so, since $N$ is finitary, it follows that $N$ embeds isomorphically in the direct sum of the various $\operatorname{Sym}_{\Gamma g} \cong \operatorname{Sym}_{n}$ where $n=|\Gamma|$. Thus $N$ is an f.c. group and $G$ has the required subnormal series.

Case 2. $k>1$

Proof. We suppose that $\Omega=\Omega_{1} \cup \Omega_{2} \cup \cdots \cup \Omega_{k}$ consists of $k$ orbits and that the result holds for $k-1$. Let $N$ be the kernel of the action of $G$ on $\Gamma=\Omega_{1} \cup \Omega_{2} \cup \cdots \cup \Omega_{k-1}$ and let $\bar{G}=G / N$. Then $\bar{G}$ acts faithfully on $\Gamma$ and certainly $\bar{G}=\bar{H}^{\bar{G}}$. Thus, since $\Gamma$ consists of $k-1$ orbits, the inductive assumption implies that $\bar{G}$ has an appropriate finite subnormal series with factors which are either f.c. groups or isomorphic to suitable finitary alternating groups FAlt .

Finally, let $\tilde{G}$ be the homomorphic image of $G$ in $\operatorname{Sym}_{\Omega_{k}}$ determined by its action on $\Omega_{k}$. Since $\tilde{G}=\tilde{H}^{\tilde{G}}$, the $k=1$ case implies that $\tilde{G}$ has a suitable subnormal series. Furthermore, $N$ acts faithfully on $\Omega_{k}$, so $N \cong \tilde{N} \triangleleft \tilde{G}$. In particular, the above series for $\tilde{G}$ determines a subnormal series for $N$ whose factors are normal subgroups of the factors of the $\tilde{G}$-series. But FAlt sim $_{\infty}$ is simple, and any subgroup of an f.c. group is also an f.c. group. Thus, we obtain an appropriate subnormal series for $N$ and, by combining it with the known series for $\bar{G}=G / N$, the result follows.

For convenience, we say that a finite group $H$ is reduced if sol $H=\langle 1\rangle$. Equivalently, $H$ is reduced if it has no nontrivial normal abelian subgroups or if $\mathbb{O}_{q}(H)=$ $\langle 1\rangle$ for all primes $q$.

Now let $H$ be an arbitrary finite group and let $\operatorname{soc} H$ be its characteristic subgroup generated by the minimal normal subgroups of $H$. Since any two distinct minimal normal subgroups commute, it follows that the socle is in fact the direct product of certain of these subgroups. Furthermore, any minimal normal subgroup of $H$ is either an elementary abelian $q$-group for some prime $q$ or it is semisimple. In particular, if $H$ is reduced, then soc $H$ is semisimple, and this proves most of 
part (ii) of the following lemma. Note that part (iv) uses a strong version of the Schreier Conjecture and requires CFSG.

Lemma 4.2. Let $H$ be a finite reduced group.

i. Any subnormal subgroup of $H$ is also reduced.

ii. The socle of $H$ is semisimple and $\mathbb{C}_{H}(\operatorname{soc} H)=\langle 1\rangle$.

iii. Write soc $H=M_{1} \times M_{2} \times \cdots \times M_{k}$ as a direct product of nonabelian simple groups. Then every automorphism of $H$ stabilizes soc $H$ and permutes the factors $M_{1}, M_{2}, \ldots, M_{k}$.

iv. If we let $N=\left\{h \in H \mid h\right.$ stabilizes all $\left.M_{i}\right\}=\bigcap_{i} \mathbb{N}_{H}\left(M_{i}\right)$, then $N^{(4)}=$ soc $H$, where $N^{(4)}$ is the fourth derived subgroup of $N$.

v. The $M_{i}$ 's above are precisely the minimal subnormal subgroups of $H$.

Proof. (i) If $S \triangleleft H$ and $q$ is any prime, then $\mathbb{O}_{q}(S)$ characteristic in $S$ implies that $\mathbb{O}_{q}(S) \subseteq \mathbb{O}_{q}(H)=\langle 1\rangle$. Thus $S$ is reduced, and the subnormal case follows easily by induction.

(ii) We already know that $\operatorname{soc} H$ is semisimple and hence it has trivial center. Thus $\mathbb{C}_{H}(\operatorname{soc} H)$ is a normal subgroup of $H$ disjoint from soc $H$, and we conclude that $\mathbb{C}_{H}(\operatorname{soc} H)=\langle 1\rangle$.

(iii) This follows since $\operatorname{soc} H$ is a characteristic subgroup of $H$ and since the factors $M_{i}$ are precisely the minimal normal subgroups of soc $H$.

(iv) If $N=\bigcap_{i} \mathbb{N}_{H}\left(M_{i}\right)$, then certainly $N \supseteq \operatorname{soc} H$ and hence $N^{(4)} \supseteq(\operatorname{soc} H)^{(4)}=$ soc $H$. On the other hand, since $\mathbb{C}_{H}(\operatorname{soc} H)=\langle 1\rangle$, we know that $N$ embeds in $\prod_{i=1}^{k} \operatorname{Aut}\left(M_{i}\right)$ with soc $H$ mapping onto $\prod_{i=1}^{k} \operatorname{Inn}\left(M_{i}\right)$. Thus $N /(\operatorname{soc} H)$ embeds in $\prod_{i=1}^{k} \operatorname{Out}\left(M_{i}\right)$, and it follows from the Classification of Finite Simple Groups and the specific computation of their automorphism groups (see [G, page 304]) that each $\operatorname{Out}\left(M_{i}\right)$ is solvable of derived length at most 4. Thus $N^{(4)} \subseteq \operatorname{soc} H$, as required.

(v) Since $M_{i} \triangleleft \triangleleft H$ and $M_{i}$ is simple, it is clear that each $M_{i}$ is a minimal subnormal subgroup of $H$. Conversely, suppose $M$ is a minimal subnormal subgroup, so that $M$ is certainly simple. If $M$ is cyclic of prime order $q$, then it follows from [Z, page 247] that $M \subseteq \mathbb{O}_{q}(H)=\langle 1\rangle$, a contradiction. Thus $M$ is nonabelian simple and [Z, page 247] implies that $\langle\operatorname{soc} H, M\rangle$ is semisimple. In particular, we know that every subnormal subgroup of the latter group is a canonical direct factor. Since this observation applies to both soc $H$ and to $M$, it follows that if $M \nsubseteq \operatorname{soc} H$, then $M \subseteq \mathbb{C}_{H}(\operatorname{soc} H)=\langle 1\rangle$, a contradiction. Thus $M \subseteq \operatorname{soc} H$, so $M \triangleleft \triangleleft \operatorname{soc} H$ and therefore $M=M_{i}$ for some $i$.

Of course, if $H$ is any finite group, then $\bar{H}=H / \operatorname{sol} H$ is reduced. Thus the above lemma applies to $\bar{H}$ and, in particular, we know that soc $\bar{H}$ is semisimple. We will be concerned with the inverse image of soc $\bar{H}$ in $H$ and, for want of a better name, we call it the radical of $H$. Thus $S=\operatorname{rad} H$ is a characteristic subgroup of $H$ 
containing sol $H$ and satisfying $S / \operatorname{sol} H=\operatorname{soc}(H / \operatorname{sol} H)$. Obviously, $\operatorname{sol} S=\operatorname{sol} H$ and $S$ is solvable-by-semisimple.

Now suppose that $G=\bigcup_{i=1}^{\infty} L_{i}$ is an ascending union of the finite groups $L_{i}$. Then each $S_{i}=\operatorname{rad} L_{i}$ is solvable-by-semisimple and part (iii) of the previous lemma implies that $L_{i}$ permutes the finite set $\Omega_{i}$ of simple factors of $S_{i} / \operatorname{sol} L_{i}$ by conjugation. As we will see, this local structure can be combined to yield a permutation representation for $G$ itself. We follow an approach suggested by recent work of [H1] and [H2] on finitary linear groups.

To start with, let $\mathcal{F}$ be an ultrafilter on the natural numbers $\mathcal{N}$ which contains all cofinite sets. Then all members of $\mathcal{F}$ are infinite and there is a natural permutation action of the ultraproduct group $L=\prod_{\mathcal{F}} L_{i}$ on the set $\Omega=\prod_{\mathcal{F}} \Omega_{i}$. Furthermore, there is a natural homomorphism $\theta: G \rightarrow L$ given by $\theta(g)=\prod_{\mathcal{F}} \theta_{i}(g)$, where $\theta_{i}(g)=g$ if $g \in L_{i}$ and $\theta_{i}(g)=1$ otherwise (see for example [KW, page 66]). In particular, the combined homomorphism $G \rightarrow L \rightarrow \operatorname{Sym}_{\Omega}$ yields a permutation representation of $G$ on $\Omega$. Of course, this action need not be faithful, but we do have some control over the kernel. Indeed, we prove

Lemma 4.3. With the above notation, if $N$ is the kernel of the action of $G$ on $\Omega$, then the fourth derived subgroup $N^{(4)}$ is locally solvable-by-semisimple.

Proof. We use the notation of the preceding paragraph. In addition, for each $i$, we let $N_{i}$ be the kernel of the action of $L_{i}$ on $\Omega_{i}$. We first show that if $g \in N$, then $\mathcal{C}(g)=\left\{i \mid \theta_{i}(g) \in N_{i}\right\}$ is contained in $\mathcal{F}$. To this end, observe that if $i \notin \mathcal{C}(g)$, then $\theta_{i}(g)$ acts nontrivially on $\Omega_{i}$ and thus we can find $\omega=\prod_{\mathcal{F}} \omega_{i} \in \Omega$ satisfying $\omega_{i} \theta_{i}(g) \neq \omega_{i}$ for all $i \in \mathcal{N} \backslash \mathcal{C}(g)$. But $g \in N$ acts trivially on $\Omega$, so

$$
\prod_{\mathcal{F}} \omega_{i}=\omega=\omega g=\prod_{\mathcal{F}} \omega_{i} \theta_{i}(g)
$$

and therefore $\mathcal{C}(g)=\left\{i \mid \omega_{i}=\omega_{i} \theta_{i}(g)\right\} \in \mathcal{F}$. Furthermore, since $\mathcal{F}$ is closed under finite intersections, we conclude more generally that if $X$ is any finite subset of $N$, then

$$
\mathcal{C}(X)=\left\{i \mid \theta_{i}(X) \subseteq N_{i}\right\}=\bigcap_{x \in X} \mathcal{C}(x) \in \mathcal{F} .
$$

Now we turn to the structure of $N^{(4)}$, and here our goal is to show that the set $\left\{N^{(4)} \cap S_{j}\right\}$ is a local system for this group. To this end, let $H$ be any finite subgroup of $N^{(4)}$ and choose $W$ to be a finite subgroup of $N$ with $H \subseteq W^{(4)}$. By the result of the previous paragraph, $\mathcal{C}(W)=\left\{i \mid \theta_{i}(W) \subseteq N_{i}\right\} \in \mathcal{F}$ and, in particular, $\mathcal{C}(W)$ is infinite. Thus there exists $j \in \mathcal{C}(W)$ with $W \subseteq L_{j}$. But then $W=\theta_{j}(W) \subseteq N_{j}$, so $H \subseteq W^{(4)} \subseteq N_{j}^{(4)} \subseteq S_{j}$ by Lemma 4.2(iv) applied to the reduced group $L_{j} /$ sol $L_{j}$. In other words, $H \subseteq N^{(4)} \cap S_{j}$ and $\left\{N^{(4)} \cap S_{j}\right\}$ is indeed a local system for $N^{(4)}$. Furthermore, since $N^{(4)} \cap S_{j} \triangleleft S_{j}$, it follows that each 
$N^{(4)} \cap S_{j}$ is solvable-by-semisimple. Finally, by deleting terms if necessary, we can write $N^{(4)}$ as the ascending union $N^{(4)}=\bigcup_{j \in \mathcal{M}} N^{(4)} \cap S_{j}$ where $\mathcal{M}$ is a suitable subsequence of $\mathcal{N}$, and consequently $N^{(4)}$ is locally solvable-by-semisimple.

We close this section by combining the various ingredients into another semiprimitivity result. Notice that neither the hypothesis nor the conclusion mentions ultraproducts.

Lemma 4.4. Let $G=\bigcup_{i=1}^{\infty} L_{i}$ be an ascending union of the finite groups $L_{i}$, and let $H$ be a subgroup of $L_{1}$ with $G=H^{G}$. Suppose that $k$ is a fixed positive integer and that, for each $i, H$ moves at most $k$ points in its conjugation action on the simple factors of $\operatorname{rad} L_{i} / \operatorname{sol} L_{i}$. If $G$ has no nonidentity locally subnormal subgroups, then $K[G]$ is semiprimitive.

Proof. We use the notation of Lemma 4.3 and the material preceding it. In particular, $\mathcal{F}$ is an ultrafilter on $\mathcal{N}$ containing all confinite subsets, and $G$ has a permutation representation ${ }^{-}: G \rightarrow \operatorname{Sym}_{\Omega}$ on the set $\Omega=\prod_{\mathcal{F}} \Omega_{i}$. Furthermore, if $N$ is the kernel of this representation, then we know from Lemma 4.3 that $N^{(4)}$ is locally solvable-by-semisimple. But $N^{(4)} \triangleleft G$, so $N^{(4)}$ has no nonidentity locally subnormal subgroups, and therefore Lemma 3.4 implies that $\mathcal{J} K\left[N^{(4)}\right]=0$.

Next, we observe that $H$ moves at most $k$ points of $\Omega$. This is, of course, a standard fact and perhaps the easiest proof starts by labeling the points of $\Omega_{i}$ moved by $H$ as $\omega_{i}^{1}, \omega_{i}^{2}, \ldots, \omega_{i}^{k}$, with duplication if $H$ moves less than $k$ points. In this way, we obtain $k$ points $\omega^{j} \in \Omega$ given by $\omega^{j}=\prod_{\mathcal{F}} \omega_{i}^{j}$, and we claim that these are the only possible points of $\Omega$ moved by $H$. Indeed, let $\alpha=\prod_{\mathcal{F}} \alpha_{i}$ be any point of $\Omega$ and define the sets $\mathcal{A}^{j}=\left\{i \mid \alpha_{i}=\omega_{i}^{j}\right\}$ for $j=1,2, \ldots, k$, and let $\mathcal{A}^{0}=\mathcal{N} \backslash \bigcup_{j=1}^{k} \mathcal{A}^{j}$. Then, since $\mathcal{F}$ is an ultrafilter and since $\bigcup_{j=0}^{k} \mathcal{A}^{j}=\mathcal{N}$, it follows that $\mathcal{A}^{j} \in \mathcal{F}$ for some $j$. But if $j \geq 1$, then $\alpha=\prod_{\mathcal{F}} \alpha_{i}=\prod_{\mathcal{F}} \omega_{i}^{j}=\omega^{j}$, whereas $j=0$ clearly implies that $H$ fixes the point $\alpha$.

Thus the claim is proved and we see that $\bar{H} \subseteq \mathrm{FSym}_{\Omega}$. In particular, since $\bar{G} \subseteq$ $\operatorname{Sym}_{\Omega}$ and $\bar{G}=\bar{H}^{\bar{G}}$, Proposition 4.1 implies that $\bar{G}=G / N$ has a finite subnormal series with factors which are either f.c. groups or isomorphic to suitable FAlt ${ }_{\infty}$. Thus the same is clearly true for the group $G / N^{(4)}$. Finally, since $\mathcal{J} K\left[N^{(4)}\right]=0$ and $G$ has no nonidentity locally subnormal subgroups, Proposition 3.1 with $G_{0}=N^{(4)}$ implies that $K[G]$ is semiprimitive.

\section{§5. Finite Wreath Products}

In some sense, Lemma 4.4 handles the semiprimitivity problem when a certain finite subgroup $H$ of $G$ moves a bounded number of points in its action on the simple factors of the various $\operatorname{rad} L_{i} / \operatorname{sol} L_{i}$. Thus it remains to consider the case where the number of moved points is unbounded, and that is the goal of this section. As will be apparent, the argument requires a close look at the irreducible representations 
of certain finite groups having structures similar to that of a wreath product. Of course, when dealing with such representations, it is usually appropriate to work over algebraically closed fields. The following lemma contains the key idea.

Lemma 5.1. Let $G=S \rtimes W$ be a finite semidirect product and let $\tilde{K}$ denote the algebraic closure of $K$. Suppose that

i. $S=S_{0} \times S_{1} \times \cdots \times S_{t}$ is a direct product of $t+1$ groups,

ii. $W$ stabilizes $S_{0}$ and permutes the remainings $S_{i}$ 's,

iii. $W$ acts faithfully as a permutation group on the set $\left\{S_{1}, S_{2}, \ldots, S_{t}\right\}$, and

iv. for each $i=1,2, \ldots, t$, the group algebra $\tilde{K}\left[S_{i}\right]$ has at least $|W|$ distinct irreducible representations.

Then $\mathcal{J} K[G] \cap K[W]=0$.

Proof. Since $\mathcal{J} K[G]$ is nilpotent, it is clear that $\mathcal{J} K[G] \subseteq \mathcal{J} \tilde{K}[G]$. Thus it suffices to show that $\mathcal{J} \tilde{K}[G] \cap \tilde{K}[W]=0$, or equivalently we can assume that $K$ is algebraically closed. Furthermore, since we will need to consider the orbits of $W$ on $\left\{S_{1}, S_{2}, \ldots, S_{t}\right\}$, it is convenient to relabel these factors using double subscripts so that $\left\{S_{i, 1}, S_{i, 2}, \ldots, S_{i, t_{i}}\right\}$ is the $i$ th orbit of the action. In particular, if $W_{i}$ is the stabilizer (normalizer) of $S_{i, 1}$ in $W$, then $\left|W: W_{i}\right|=t_{i}$ and we can choose right coset representatives $\left\{w_{i, 1}, w_{i, 2}, \ldots, w_{i, t_{i}}\right\}$ so that $S_{i, j}=S_{i, 1}^{w_{i, j}}$.

Now $W_{i}$ normalizes $S_{i, 1}$ and therefore it permutes the irreducible representations of $K\left[S_{i, 1}\right]$. But, by assumption (iv), there are at least $|W|$ of the latter, so there are at least $|W| /\left|W_{i}\right|=t_{i}$ orbits of these representations. In particular, we can let $\phi_{i, 1}, \phi_{i, 2}, \ldots, \phi_{i, t_{i}}$ be irreducible representations of $K\left[S_{i, 1}\right]$ in distinct $W_{i}$-orbits, and we define the irreducible representation $\theta_{i, j}$ of $K\left[S_{i, j}\right]$ by $\theta_{i, j}=\phi_{i, j}^{w_{i, j}}$. For convenience, let $\theta_{0}$ denote the principal representation of $K\left[S_{0}\right]$.

Since $K$ is algebraically closed, it now follows that

$$
\theta=\theta_{0} \otimes\left(\bigotimes_{i, j} \theta_{i, j}\right)
$$

is an irreducible representation for

$$
K[S]=K\left[S_{0}\right] \otimes\left(\bigotimes_{i, j} K\left[S_{i, j}\right]\right)
$$

Of course, $W$ normalizes $S$, so $W$ permutes the irreducible representations of $K[S]$, and we claim that it acts regularly on the $W$-orbit of $\theta$.

Indeed, suppose $w \in W$ stabilizes $\theta$ and let $i, i^{\prime}, j, k$ be subscripts with $S_{i, j}^{w}=$ $S_{i^{\prime}, k}$. Then $S_{i, j}$ and $S_{i^{\prime}, k}$ are in the same $W$-orbit, so clearly $i^{\prime}=i$. Furthermore, since $\theta^{w}=\theta$, uniqueness of tensor factors implies that $\theta_{i, j}^{w}=\theta_{i, k}$. Thus,

$$
\phi_{i, j}^{w_{i, j} w}=\theta_{i, j}^{w}=\theta_{i, k}=\phi_{i, k}^{w_{i, k}}
$$


or equivalently $\phi_{i, j}^{w_{i, j} w w_{i, k}^{-1}}=\phi_{i, k}$. But $w_{i, j} w w_{i, k}^{-1} \in W_{i}$, so $\phi_{i, j}$ and $\phi_{i, k}$ are in the same $W_{i}$-orbit and therefore $j=k$. In other words, we have shown that $w$ acts trivially as a permutation on $\left\{S_{i, j}\right\}$, so hypothesis (iii) implies that $w=1$.

Finally, since $G=S W=S \rtimes W$, we now know that the induced representation

$$
\theta^{G}=\theta \otimes_{K[S]} K[G]=\theta \otimes_{K} K[W]
$$

is irreducible and hence $\theta^{G}(\mathcal{J} K[G])=0$. On the other hand, the restriction $\left(\theta^{G}\right)_{W}$ is isomorphic to the direct sum of $\theta(1)$ copies of the regular representation of $K[W]$, and therefore $\theta^{G}$ is faithful on $K[W]$. In particular, since $\theta^{G}(\mathcal{J} K[G] \cap K[W])=0$, we conclude that $\mathcal{J} K[G] \cap K[W]=0$, as required.

We remark that the assumption above on the number of irreducible representations of each $\tilde{K}\left[S_{i}\right]$ is definitely required. This can be seen in Example 5.3 below. To start with, we need

Lemma 5.2. Let $W$ be a finite group and let $R * W$ be a skew group ring which is a K-algebra over a field of characteristic $p>0$. Suppose $1=e_{1}+e_{2}+\cdots+e_{t}$ is an orthogonal decomposition of 1 into central idempotents of $R$ and that $W$ permutes the set $\left\{e_{1}, e_{2}, \ldots, e_{t}\right\}$. Furthermore, if $W_{i}$ is the stabilizer of $e_{i}$ in $W$, assume that $\left|W_{i}\right|$ is divisible by $p$ and that some subgroup $P_{i}$ of $W_{i}$ of order $p$ centralizes $\operatorname{Re}_{i}$. Then $\hat{W} \in \mathcal{J}(R * W) \cap K[W]$, where $\hat{W}=\sum_{w \in W} w$ is the sum of the elements of $W$ in $K[W] \subseteq R * W$.

Proof. If $X$ is any subset of $W$, we let $\hat{X}$ denote the sum of the elements of $X$ in $K[W]$. Note that if $w \in W$, then $w \hat{W}=\hat{W}=\hat{W} w$ and $w R=R w$. Thus, it follows that $e_{i} \hat{W} R$ is a right ideal of $R * W$, and we claim that this ideal has square zero. For this, it suffices to show that

$$
0=e_{i} \hat{W} R e_{i} \hat{W}=e_{i} \hat{W} e_{i} R \hat{W} .
$$

To this end, observe that if $w \in W$, then $e_{i} w e_{i}=w e_{i}^{w} e_{i}=w e_{j} e_{i}$, where $e_{i}^{w}=$ $e_{j}$. Thus this product is zero if $w \notin W_{i}$, and it is equal to $w e_{i}$ when $w \in W_{i}$. Consequently, $e_{i} \hat{W} e_{i}=\hat{W}_{i} e_{i}$, and hence

$$
e_{i} \hat{W} e_{i} R \hat{W}=\hat{W}_{i}\left(e_{i} R\right) \hat{W} .
$$

Furthermore, note that $\hat{W}_{i}=\hat{C}_{i} \hat{P}_{i}$, where $C_{i}$ is a set of left coset representatives for $P_{i}$ in $W_{i}$ and, since $P_{i}$ centralizes $e_{i} R$, we have

$$
\hat{W}_{i}\left(e_{i} R\right) \hat{W}=\hat{C} \hat{P}_{i}\left(e_{i} R\right) \hat{W}=\hat{C}_{i}\left(e_{i} R\right) \hat{P}_{i} \hat{W} .
$$

But $\hat{P}_{i} \hat{W}=\left|P_{i}\right| \hat{W}=p \hat{W}=0$, so the claim is proved.

It follows that $e_{i} \hat{W} R \subseteq \mathcal{J}(R * W)$, so $e_{i} \hat{W} \in \mathcal{J}(R * W)$ for each $i$ and hence we have $\hat{W}=\sum_{i=1}^{t} e_{i} \hat{W} \in \mathcal{J}(R * W)$, as required.

With this, we can prove 
Example 5.3. Let $A$ be a finite abelian group with precisely $m$ irreducible representations over the algebraically closed field $K$ of characteristic $p>0$ and let $t=m(p-1)+1$. If $G$ is the permutation wreath product $G=A \imath \operatorname{Sym}_{t}=S \rtimes W$, where $S=A \times A \times \cdots \times A$ is the base group and $W=\operatorname{Sym}_{t}$, then $\hat{W} \in \mathcal{J} K[G] \cap K[W]$. Hence, the latter intersection is nonzero.

Proof. Since $S \triangleleft G$, we have $\mathcal{J} K[S] \cdot K[G] \subseteq \mathcal{J} K[G]$ and hence it suffices to $\bmod$ out by this ideal. When we do so, we obtain a skew group ring $R * W$, where $R$ is the semisimple $K$-algebra $K[S] / \mathcal{J} K[S]$. In fact, $R=R_{1} \otimes R_{2} \otimes \cdots \otimes R_{t}$, where each $R_{i}$ is isomorphic to the commutative semisimple algebra $K[A] / \mathcal{J} K[A]$. Furthermore, $W=\operatorname{Sym}_{t}$ permutes the $R_{i}$ 's via the natural action.

Note that the irreducible representations of $R$ are all tensor products of the form $\theta=\theta_{1} \otimes \theta_{2} \otimes \cdots \otimes \theta_{t}$, where each $\theta_{i}$ is an irreducible representation of $R_{i} \cong K[A] \mathcal{J} K[A]$. Furthermore, $K[A] / \mathcal{J} K[A]$ has precisely $m$ distinct irreducible representations and $t>m(p-1)$. Thus, for any such $\theta$, at least $p$ of the $\theta_{i}$ are identical, and it follows that $\theta$ is fixed by a $p$-cycle $w_{\theta} \in W=\operatorname{Sym}_{t}$. In particular, if $e_{\theta}$ is the centrally primitive idempotent of $R$ corresponding to $\theta$, then $w_{\theta}$ centralizes $e_{\theta}$. In fact, since $R$ is commutative, we have $e_{\theta} R=e_{\theta} K$ and hence $w_{\theta}$ centralizes $e_{\theta} R$. Lemma 5.2 now implies that $\hat{W} \in \mathcal{J}(R * W) \cap K[W]$ and, by pulling back this inclusion, we obtain $\hat{W} \in \mathcal{J} K[G] \cap K[W]$.

The preceding example is somewhat unsatisfactory in terms of the type of group we are studying here. Specifically, we are interested in groups of the form $G=$ $S \rtimes W$ where $S$ is semisimple, rather than abelian. Unfortunately, we do not have a construction which can handle that situation.

Hypothesis (iv) of Lemma 5.1 also gives rise to another natural question. Namely, what can be said about a finite group $H$ if we know the number $m$ of irreducible representations of $K[H]$ where $K$ is an algebraically closed field? If char $K=0$, there is an old result (see [I2, Theorem 4.13]) which asserts that $|H|$ is bounded by a function of $m$. On the other hand, if char $K=p>0$, then the answer is not so obvious. To start with, the number of such representations is the same as the number of $p$-regular classes of $H$ (see [I1, Corollary 15.11]). Thus, for example, if $H$ is a $p$-group, then $m=1$ while $|H|$ can be arbitrarily large.

A next guess might be that $\left|H / \mathbb{O}_{p}(H)\right|$ is bounded by a function of $m$, but this is most likely also false. For example, if $p=2^{n}-1$ is a Mersenne prime, then we can let $H=A \rtimes P$ where $A$ is an elementary abelian 2-group of order $2^{n}, P$ is cyclic of order $p$, and $P$ acts transitively on the nonidentity elements of $A$. Then $\mathbb{O}_{p}(H)=\langle 1\rangle$ and $m=2$, while $|H|=p(p+1)$ can presumably be unbounded. In general, it is possible that if both $\mathbb{O}_{p}(H)=\langle 1\rangle$ and $\mathbb{O}^{p^{\prime}}(H)=H$, then $|H|$ is bounded by a function of $m$.

For our purposes, it is more appropriate to assume that $H$ is nonabelian and simple. Here $|H|$ is probably bounded by a function of $m$ using a proof based on CFSG. For example, suppose $H=\operatorname{Alt}_{n}$. If $p \neq 3$, then $H$ has $p$-regular classes 
consisting of products of $t$ disjoint 3 -cycles for $t=0,1, \ldots,[n / 3]$. Hence $1+[n / 3] \leq$ $m$ and $n \leq 3 m$. On the other hand, if $p=3$, then $H$ has $p$-regular classes consisting of products of $2 t$ disjoint 2 -cycles for $t=0,1, \ldots,[n / 4]$, and hence $n \leq 4 m$. Thus, in either case, $|H|=n ! / 2<(4 m)$ !.

For the classical linear groups, one can use their order formulas (see [G, page 135]) along with the Miraculous Prime Theorem ([Ar, Corollary 1]) to guarantee that if the rank of $H$ is large, then $|H|$ is divisible by many distinct primes. But $m$ clearly bounds the number of distinct prime factors of $|H|$, and therefore it also bounds the rank of $H$. This solves half the problem, but there still remains the task of bounding the size of the defining field for all finite simple groups of Lie type. This appears to be a more tedious problem, but certainly it can be settled.

In the case of semisimple groups, if $S=M_{1} \times M_{2} \times \cdots \times M_{k}$ is a direct product of $k$ nonabelian simple groups, then each $K\left[M_{i}\right]$ has at least 2 irreducible representations and therefore, by taking tensor products, $K[S]$ has at least $2^{k}$ irreducible representations. In particular, $k \leq \log _{2} m$, and this elementary fact is all we require.

Lemma 5.4. Let $G=S W$ be a finite group with $S$ a normal semisimple subgroup. Suppose that, under the permutation action of $W$ on the simple factors of $S$, every nonidentity normal subgroup $N$ of $W$ moves at least $4^{|W|}$ points. Then it follows that $\mathcal{J} K[G] \cap K[W]=0$.

Proof. First observe that $S \cap W$ is a normal subgroup of $W$ which normalizes all simple factors of $S$. Thus, by assumption, $S \cap W=\langle 1\rangle$ and $G=S W=S \rtimes W$ is a semidirect product.

Next, we partition the simple factors of $S$ into larger subgroups $S_{i, j}$ via the following somewhat complicated procedure. To start with, let $W_{1}, W_{2}, \ldots, W_{t}$ be representatives of the conjugacy classes of subgroups of $W$ and, for each $i$, let $\left\{1=w_{i, 1}, w_{i, 2}, \ldots, w_{i, n_{i}}\right\}$ be a full set of right coset representatives for $W_{i}$ in $W$. Of course, every transitive permutation representation $(W, \Gamma)$ of $W$ is isomorphic to a unique $\left(W, W / W_{i}\right)$, where the latter is the permutation action of $W$ on the cosets of $W_{i}$.

Now $W$ permutes the set $\Omega$ of simple factors of $S$ by conjugation and, for each $i$, let $\Omega_{i, 1}, \Omega_{i, 2}, \ldots, \Omega_{i, v_{i}}$ be the orbits of this action which are isomorphic to $\left(W, W / W_{i}\right)$. In particular, for each $1 \leq k \leq v_{i}$, we can choose a, not necessarily unique, simple factor $M_{i, k} \in \Omega_{i, k}$ whose stabilizer in $W$ is equal to $W_{i}$. Then $\Omega_{i, k}=$ $\left\{M_{i, k}^{w_{i, j}} \mid j=1,2, \ldots, n_{i}\right\}$ and, for each $i, j$, we define $S_{i, j}=\prod_{k=1}^{v_{i}} M_{i, k}^{w_{i, j}} \triangleleft S$. Note that

1. $S$ is the direct product $S=\prod_{i=1}^{t} \prod_{j=1}^{n_{i}} S_{i, j}$,

2. $S_{i, 1}$ is stabilized by $W_{i}$ and $S_{i, j}=S_{i, 1}^{w_{i, j}}$,

3. $S_{i, j}$ contains at most one simple factor from each $W$-orbit of $\Omega$.

Furthermore, if $\mathcal{V}=\left\{i\left|v_{i} \geq \log _{2}\right| W \mid\right\}$ and if $S_{0}=\prod_{i \notin \mathcal{V}} \prod_{j=1}^{n_{i}} S_{i, j}$, then

4. $S$ is the direct product $S=S_{0} \times \prod_{i \in \mathcal{V}} \prod_{j=1}^{n_{i}} S_{i, j}$. 
It is now clear that $G=S \rtimes W$ satisfies most of the hypotheses of Lemma 5.1. Indeed, (i) and (ii) follow from (4) and (2), respectively, while (iv) follows from the fact that each $S_{i, j}$ with $i \in \mathcal{V}$ is a direct product of $v_{i} \geq \log _{2}|W|$ nonabelian simple groups. Finally, suppose $N \triangleleft W$ is the kernel of the permutation action of $W$ on $\left\{S_{i, j} \mid i \in \mathcal{V}, 1 \leq j \leq n_{i}\right\}$. Then $N$ normalizes each $S_{i, j}$ with $i \in \mathcal{V}$ and hence it permutes the simple factors which generate these groups. But, by (3), $S_{i, j}$ contains at most one simple factor from each $W$-orbit of $\Omega$, and therefore it follows that $N$ must stabilize each such point. In other words, the only simple factors moved by $N$ are those contained in $S_{0}$. But observe that $t \leq 2^{|W|-1}$ and $n_{i}=\left|W: W_{i}\right| \leq|W|$. Thus, since $S_{0}$ is generated by those $S_{i, j}$ which contain $v_{i}<\log _{2}|W|$ simple factors, we conclude that $S_{0}$ contains at most

$$
\left(\log _{2}|W|\right) \cdot|W| \cdot 2^{|W|-1}<|W|^{2} \cdot 2^{|W|-1}<2^{|W|+1} \cdot 2^{|W|-1}=4^{|W|}
$$

points of $\Omega$. Consequently, $N$ moves less than $4^{|W|}$ points of $\Omega$ and therefore, by assumption, $N=\langle 1\rangle$.

Thus $G=S \rtimes W$ satisfies all the hypotheses of Lemma 5.1, and we conclude from the latter result that $\mathcal{J} K[G] \cap K[W]=0$.

\section{$\S 6$. The Main Theorem}

In this final section, we prove our main results on semiprimitivity. To start with, we offer the

Proof of Theorem 1.3. Recall that $G$ is assumed to be a locally finite group having no nonidentity locally subnormal subgroups and that $K$ is a field of characteristic $p>0$. Our aim is to show that $K[G]$ is semiprimitive and we suppose, by way of contradiction, that this is not the case. Then, by Lemmas 1.1 and 2.5, there exists a countable subgroup $\tilde{G}$ of $G$ such that $\tilde{G}$ has no nonidentity locally subnormal subgroups and $\mathcal{J} K[\tilde{G}] \neq 0$. In other words, $\tilde{G}$ is also a counterexample, so it suffices to assume that $G=\tilde{G}$ is countable and, in particular, that $G$ is the ascending union of the finite groups $L_{1} \subseteq L_{2} \subseteq L_{3} \subseteq \cdots$.

Since $\mathcal{J} K[G] \neq 0$, there exists an element $0 \neq \alpha \in \mathcal{J} K[G]$ and finite subgroups $\langle\operatorname{supp} \alpha\rangle=H \subseteq W$ which satisfy the conclusions of Lemma 2.4. For example, part (iv) of that lemma implies that if $T$ is any subgroup of $G$ normalized by $W$ and if $\mathcal{J} K[T]=0$, then $W \subseteq \Delta(T W)$. By deleting a few terms if necessary, we can of course assume that $W \subseteq L_{1}$.

Now, if $S_{i}=\operatorname{rad} L_{i}$, then $W$ permutes the set $\Omega_{i}$ of simple factors of $S_{i} / \operatorname{sol} L_{i}$ by conjugation and, for each nonidentity normal subgroup $N$ of $W$, we let $f_{N}(i)$ be the number of points of $\Omega_{i}$ moved by $N$. In this way, we obtain a finite collection of functions $f_{N}: \mathcal{N} \rightarrow \mathcal{N} \cup\{0\}$ and, as usual, there are two cases to consider according to whether these functions are bounded or not. 
Case 1. For some $\langle 1\rangle \neq N \triangleleft W$, the map $f_{N}$ is bounded on a subsequence of $\mathcal{N}$.

Proof. By deleting terms, we can assume that $f_{N}$ is bounded on $\mathcal{N}$. Let $k$ be an upper bound for the values of $f_{N}$, and let $T=N^{[G]}$ be the local subnormal closure of $N$ in $G$. We apply the various conclusions of Lemma 2.2. To start with, (iii) shows that $T$ has no nonidentity locally subnormal subgroups, and (iv) implies that $W$ normalizes $T$ and that $T=N^{T}$. Furthermore, by part (ii), $\left\{N^{\left[L_{i}\right]} \mid i=1,2, \ldots\right\}$ is a local system for $T$ and, by deleting terms if necessary, we can assume that $T$ is the ascending union of the subgroups $T_{i}=N^{\left[L_{i}\right]} \triangleleft \triangleleft L_{i}$.

Since $T_{i} \triangleleft \triangleleft L_{i}$, we know that

$$
T_{i} / \operatorname{sol} T_{i}=T_{i} /\left(T_{i} \cap \operatorname{sol} L_{i}\right) \cong T_{i}\left(\operatorname{sol} L_{i}\right) / \operatorname{sol} L_{i} \triangleleft \triangleleft L_{i} / \operatorname{sol} L_{i} .
$$

In particular, any minimal subnormal subgroup of $T_{i} / \operatorname{sol} T_{i}$ is also a minimal subnormal subgroup of $L_{i} / \operatorname{sol} L_{i}$, so it follows from Lemma 4.2(v) that $\operatorname{rad} T_{i} / \operatorname{sol} T_{i}$ is a direct factor of $\operatorname{rad} L_{i} / \operatorname{sol} L_{i}$. More precisely, if $\Gamma_{i}$ denotes the set of simple factors of $\operatorname{rad} T_{i} / \operatorname{sol} T_{i}$, then $\Gamma_{i} \subseteq \Omega_{i}$. Indeed, since this inclusion respects the action of $N$, we see that $N$ moves at most $k$ points of $\Gamma_{i}$.

Finally, since $T=N^{T}$, Lemma 4.4 implies that $\mathcal{J} K[T]=0$. Therefore, by Lemma 2.4, we conclude that $W \subseteq \Delta(T W)$ and hence that $N \subseteq T \cap \Delta(T W) \subseteq$ $\Delta(T)$. But $\Delta(T)$ is generated by all finite normal subgroups of $T$, so $\Delta(T)=\langle 1\rangle$ and this is a contradiction since $N \neq\langle 1\rangle$.

Case 2. The functions $f_{N}$ are unbounded on all subsequences of $\mathcal{N}$.

Proof. By [PZ, Lemma 1.3], there exists a subsequence $\mathcal{M} \subseteq \mathcal{N}$ such that each $f_{N}$ is strictly increasing on $\mathcal{M}$. Consequently, $\min _{N}\left\{f_{N}\right\}$ is unbounded on $\mathcal{M}$, so there exists a subscript $i$ with $f_{N}(i) \geq 4^{|W|}$ for all $N$. In particular, since $S_{i} \cap W \triangleleft W$ stabilizes all simple factors of $S_{i} /$ sol $L_{i}$, it follows that $S_{i} \cap W=\langle 1\rangle$. Now let $G_{i}=S_{i} W=S_{i} \rtimes W$ and consider the natural epimorphism ${ }^{-}: G_{i} \rightarrow$ $G_{i} /$ sol $S_{i}$. Then $\bar{G}_{i}=\bar{S}_{i} \rtimes \bar{W}$, where $\bar{S}_{i}=S_{i} / \operatorname{sol} S_{i}$ and $\bar{W} \cong W$, so Lemma 5.4 and $f_{N}(i) \geq 4^{|W|}$ imply that $\mathcal{J} K\left[\bar{G}_{i}\right] \cap K[\bar{W}]=0$. On the other hand, $\alpha \in K[W]$ and $\alpha \in \mathcal{J} K[G] \cap K\left[G_{i}\right] \subseteq \mathcal{J} K\left[G_{i}\right]$, by Lemma 1.1. Thus $0 \neq \alpha \in \mathcal{J} K\left[G_{i}\right] \cap K[W]$ and hence $\bar{\alpha} \in \mathcal{J} K\left[\bar{G}_{i}\right] \cap K[\bar{W}]$. But the map ${ }^{-}: K[W] \rightarrow K[\bar{W}]$ is an isomorphism, so $\bar{\alpha} \neq 0$ and this contradiction yields the result.

As we mentioned earlier, the conjecture here is that $K[G]$ is semiprimitive if and only if $G$ has no locally subnormal subgroups of order divisible by $p$. Thus Theorem 1.3 shows that we are on the right track, and so also does the following special case.

Corollary 6.1. Let $G$ be a locally finite group having a local system $\mathcal{L}$ consisting of reduced groups. If char $K=p>0$, then the group algebra $K[G]$ is semiprimitive if and only if $G$ has no locally subnormal subgroups of order divisible by $p$. 
Proof. Let $K$ be a field of characteristic $p>0$ and let $G$ be a locally finite group. If $G$ has a locally subnormal subgroup of order divisible by $p$, then $\mathcal{J} K[G] \neq 0$ by Lemma 2.1(ii). For the reverse implication, we assume that $G$ has a local system $\mathcal{L}$ consisting of reduced groups and that $G$ has no locally subnormal subgroups of order divisible by $p$. The goal is to show that $K[G]$ is semiprimitive.

To start with, let $S$ be the characteristic subgroup of $G$ generated by the set $\Gamma=\left\{M_{i} \mid i \in \mathcal{I}\right\}$ of minimal locally subnormal subgroups of $G$. If $M_{i}$ and $M_{j}$ are two such generators and if $M_{i}, M_{j} \subseteq L$ for some $L \in \mathcal{L}$, then $M_{i}$ and $M_{j}$ are clearly minimal subnormal subgroups of $L$. Thus, since $L$ is reduced, Lemma $4.2(\mathrm{v})$ implies that $M_{i}$ and $M_{j}$ are nonabelian simple and that $\left\langle M_{i}, M_{j}\right\rangle=M_{i} \times M_{j}$. It follows that $S=M_{1} \times M_{2} \times M_{3} \times \cdots$ is the direct product of the members of $\Gamma$, and hence that $\mathbb{Z}(S)=\langle 1\rangle$. Furthermore, by assumption, $S$ is a $p^{\prime}$-group and therefore we have $\mathcal{J} K[S]=0$.

Now let $C=\mathbb{C}_{G}(S)$ and $D=\mathbb{D}_{G}(S)$. Since $C \cap S=\mathbb{Z}(S)=\langle 1\rangle$ and $S$ contains all the minimal locally subnormal subgroups of $G$, it follows that $C$ has no nonidentity locally subnormal subgroups. Furthermore, since $D \triangleleft G$, we know that $D$ has no locally subnormal subgroups of order divisible by $p$. Finally, $\mathcal{J} K[S]=0$ and Lemma 2.3 imply that $\mathcal{J} K[G]=\mathcal{J} K[D] \cdot K[G]$. Thus, our goal is to prove that $\mathcal{J} K[D]=0$.

Suppose, by way of contradiction, that $\mathcal{J} K[D] \neq 0$. Then we can choose $0 \neq$ $\alpha \in \mathcal{J} K[D]$, and we set $H=\langle\operatorname{supp} \alpha\rangle$ and $T=H^{[D]}$, the local subnormal closure of $H$ in $D$. Of course, $\alpha \in \mathcal{J} K[D] \cap K[T] \subseteq \mathcal{J} K[T]$, so $K[T]$ is not semiprimitive. Furthermore, Lemma 2.2 implies that $T$ has no locally subnormal subgroups of order divisible by $p$ and that $T=H^{T}$ is the normal closure of $H$ in $T$.

Now $\mathbb{C}_{T}(S)=C \cap T \triangleleft T$ and we note that if $A$ lsn $(C \cap T)$, then $A$ lsn $T$ and therefore $A \operatorname{lsn} G$. But then $A$ lsn $C$, and this yields $A=\langle 1\rangle$. In other words, $C \cap T$ satisfies all the hypotheses of Theorem 1.3, and therefore we conclude from our main theorem that $\mathcal{J} K[C \cap T]=0$.

Next, note that $T$ permutes the simple factors $M_{i}$ of $S$ and therefore it permutes the elements of $\Omega=\bigcup_{i \in \mathcal{I}} M_{i} \subseteq S$. Indeed, since $T \subseteq D$, each element $x \in T$ centralizes a subgroup of $S$ of finite index, and therefore a normal subgroup of $S$ of finite index. But all normal subgroups of $S$ are partial products of the $M_{i}$ 's, so it follows that $x$ centralizes all but finitely many of the $M_{i}$ 's. In other words, $x$ acts like a finitary permutation on $\Omega$. Of course, the kernel of the action of $T$ on $\Omega$ is $C \cap T$, so we conclude that $\bar{T}=T /(C \cap T) \subseteq$ FSym $_{\Omega}$. Thus, since $\bar{T}=\bar{H}^{\bar{T}}$, Proposition 4.1 implies that $\bar{T}$ has a finite subnormal series with factors which are either f.c. groups or isomorphic to suitable FAlt .

Finally, by lifting the above series, we see that $T$ has a subnormal series

$$
C \cap T=T_{0} \triangleleft T_{1} \triangleleft \cdots \triangleleft T_{n}=T
$$

with each quotient $T_{i+1} / T_{i}$ either infinite simple or generated by locally subnormal subgroups. Thus, since $\mathcal{J} K\left[T_{0}\right]=\mathcal{J} K[C \cap T]=0$ and since $T$ has no locally 
subnormal subgroups of order divisible by $p$, Proposition 3.1 implies that $K[T]$ is semiprimitive. But this contradicts the fact that $0 \neq \alpha \in \mathcal{J} K[T]$, and therefore the corollary is proved.

The above argument actually offers an outline as to how to proceed further. Namely, assume that $G$ has no locally subnormal subgroups of order divisible by $p$, and let $S$ be the characteristic subgroup of $G$ generated by all its locally subnormal subgroups. Then $S$ is a $p^{\prime}$-group, so $\mathcal{J} K[S]=0$ and therefore it suffices to prove that $K\left[\mathbb{D}_{G}(S)\right]$ is semiprimitive. For this, we must first consider $\mathbb{C}_{G}(S)$ and then the factor group $\mathbb{D}_{G}(S) / \mathbb{C}_{G}(S)$. Since both of these tasks will surely require additional work and additional ideas, they are best left for later projects. In fact, once those tasks are completed, we are still left with the problem of describing $\mathcal{J} K[G]$ for arbitrary locally finite groups $G$, and that will offer some additional challenges.

\section{REFERENCES}

[Am] S. A. Amitsur, On the semi-simplicity of group algebras, Mich. Math. J. 6 (1959), 251-253.

[Ar] E. Artin, The orders of the linear groups, Comm. Pure Applied Math. 8 (1955), 355-366.

[BP] B. Bruno and R. E. Phillips, Residual properties of finitary linear groups, J. Algebra 166 (1994), 379-392.

[G] D. Gorenstein, Finite Simple Groups, Plenum, New York, 1982.

[H1] J. I. Hall, Locally finite simple groups of finitary linear transformations, Conference report, Istanbul, Turkey (to appear).

[H2] - Periodic simple groups of finitary linear transformations (to appear).

[I1] I. M. Isaacs, Character Theory of Finite Groups, Academic Press, New York, 1976.

[I2] Algebra: A Graduate Course, Brooks/Cole, Pacific Grove, 1994.

[KW] O. H. Kegel and B. A. F. Wehrfritz, Locally Finite Groups, North-Holland, Amsterdam, 1973.

[P1] D. S. Passman, A new radical for group rings?, J. Algebra 28 (1974), 556-572.

[P2] - The Algebraic Structure of Group Rings, Wiley-Interscience, New York, 1977.

[P3] , The Jacobson radical of a group ring of a locally solvable group, Proc. London Math. Soc. 38 (1979), 169-192, (ibid 39 (1979), 208-210).

[P4] Semiprimitivity of group algebras of locally finite groups, Infinite Groups and Group Rings, World Scientific, Singapore, 1993, pp. 77-101.

[P5] , Semiprimitivity of group algebras of infinite simple groups of Lie Type, Proc. Amer. Math. Soc. 121 (1994), 399-403.

[P6] _ Semiprimitivity of group algebras of locally finite groups II, J. Pure Appl. Algebra (to appear).

[P7] - Semiprimitivity of group algebras: a survey (to appear).

[PZ] D. S. Passman and A. E. Zalesskii, Semiprimitivity of group algebras of locally finite simple groups, Proc. London Math. Soc. 67 (1993), 243-276.

[W1] H. Wielandt, Eine Verallgemeinerung der invarianten Untergruppen, Math. Zeit. 45 (1939), 209-244.

[W2] - Unendliche Permutationsgruppen, Lecture Notes, Mathematisches Institut der Universität, Tübingen, 1960.

[W3] - Mathematical Works, Volume 1, de Gruyter, Berlin, 1994.

[Z] H. Zassenhaus, The Theory of Groups, Second Edition, Chelsea, New York, 1958.

Department of Mathematics, University of Wisconsin, Madison, Wisconsin 53706 
E-mail address: passman@math.wisc.edu 